\title{
Cross sections for pentaquark baryon production from protons in reactions induced by hadrons and photons
}

\author{
W. Liu and C. M. Ko \\ Cyclotron Institute and Physics Department, Texas A\&M University, College Station, Texas 77843-3366, USA
}

(Received 13 August 2003; published 8 October 2003)

\begin{abstract}
Using hadronic Lagrangians that include the interaction of pentaquark $\Theta^{+}$baryon with $K$ and $N$, we evaluate the cross sections for its production from meson-proton, proton-proton, and photon-proton reactions near threshold. With empirical coupling constants and form factors, the predicted cross sections are about $1.5 \mathrm{mb}$ in kaon-proton reactions, $0.1 \mathrm{mb}$ in $\rho$-nucleon reactions, $0.05 \mathrm{mb}$ in pion-nucleon reactions, $20 \mu \mathrm{b}$ in protonproton reactions, and $40 \mathrm{nb}$ in photon-proton reactions.
\end{abstract}

DOI: 10.1103/PhysRevC.68.045203 PACS number(s): 13.75.Gx, 13.75.Jz, 12.39.Mk, 14.20.-c

\section{INTRODUCTION}

Recently, a narrow baryon state was inferred from the invariant mass spectrum of $K^{+} n$ or $K^{0} p$ in nuclear reactions induced by photons [1,2] or kaons [3]. The extracted mass of about $1.54 \mathrm{GeV}$ and width of less than $21-25 \mathrm{MeV}$ are consistent with those of the pentaquark baryon $\Theta^{+}$consisting of $u u d d \bar{s}$ quarks predicted in the chiral soliton model [4]. Its existence has also been verified recently in the constituent quark model [5,6] and the QCD sum rules [7]. Although the spin and isospin of $\Theta^{+}$are predicted to be $1 / 2$ and 0 , respectively, those of the one detected in experiments are not yet determined. Studies have therefore been carried out to predict its decay branching ratios based on different assignments of its spin and isospin [8,9]. Since both kaons and nucleons are present in the hadronic matter formed in relativistic heavy ion collisions, the number of $\Theta^{+}$produced in these collisions may be appreciable. Using the statistical model, which assumes that $\Theta^{+}$baryons are in chemical equilibrium with other hadrons, Randrup [10] has estimated its abundance and has found that the $\Theta / \Lambda$ ratio in the midrapidity is about $(10-20) \%$ in central $\mathrm{Au}+\mathrm{Au}$ collisions at $\sqrt{s_{N N}}$ $=200 \mathrm{GeV}$ available from the Relativistic Heavy Ion Collider. With about seven $\Lambda$ 's produced in midrapidity $[11,12]$, one expects that there would be about one midrapidity $\Theta^{+}$ present in these collisions. Since a quark-gluon plasma is believed to have formed in the initial stage of relativistic heavy ion collisions, $\Theta^{+}$baryons can also be produced during the hadronization of the quark-gluon plasma. This contribution has been studied in Ref. [13] using the quark coalescence model and was found to be important as production from later hadronic matter is less significant due to the small coupling between $\Theta^{+}$and other hadrons as predicted in the chiral soliton model [4]. The coupling may even be smaller as reanalysis of $K^{+} p$ and $K^{+} d$ elastic scattering data has indicated that they are consistent with the existence of resonances with width of only $1 \mathrm{MeV}$ [14]. In this case, $\Theta^{+}$ baryon, like other multistrange baryons [15], can also be used as a signal for the quark-gluon plasma in relativistic heavy ion collisions. On the other hand, if the $\Theta^{+}$baryon interacts strongly in hadronic matter, its final number in relativistic heavy ion collisions would become independent of its initial number produced from the quark-gluon plasma.
Therefore, knowledge of the cross sections for $\Theta^{+}$production and absorption by hadrons is important for understanding the mechanism for its production in relativistic heavy ion collisions.

In this paper, we evaluate the cross sections for $\Theta^{+}$production from nucleons induced by mesons and protons using a hadronic model that is based on SU(3) flavor symmetry with empirical hadron masses and form factors at interaction vertices. For the coupling constant between $\Theta^{+}$and $K N$, it is determined from the width of $\Theta^{+}$. Introducing the photon as a $U_{e m}(1)$ gauge boson, we extend the hadronic model to also calculate the cross section for $\Theta^{+}$production from photonproton reactions, which is relevant to the experiments in which $\Theta^{+}$was detected $[1,2]$.

This paper is organized as follows. In Sec. II, the cross sections for $\Theta^{+}$production from meson-nucleon reactions are evaluated. Production of $\Theta^{+}$from proton-proton reactions is studied in Sec. III with inclusion of both two-body and threebody final states. The cross section for $\Theta^{+}$production from photon-proton reactions is then determined in Sec. IV. Finally, a summary is given in Sec. V.

\section{II. @ PRODUCTION FROM MESON-NUCLEON REACTIONS}

Possible reactions for $\Theta^{+}$production in meson-nucleon interactions are $\pi N \rightarrow \bar{K} \Theta, \quad K N \rightarrow \pi \Theta$, and $\rho N \rightarrow \bar{K} \Theta$ as shown by diagrams in Fig. 1. The interaction Lagrangians needed to evaluate the cross sections for these reactions are

$$
\begin{gathered}
\mathcal{L}_{K N \Theta}=i g_{K N \Theta}\left(\bar{\Theta} \gamma_{5} N \bar{K}+\bar{N} \gamma_{5} \Theta K\right), \\
\mathcal{L}_{\pi N N}=-i g_{\pi N N} \bar{N} \gamma_{5} \pi N, \\
\mathcal{L}_{\rho N N}=g_{\rho N N} \bar{N}\left(\gamma^{\mu} \rho+\frac{\kappa_{\rho}}{2 m_{N}} \sigma^{\mu \nu} \partial_{\mu} \rho_{\nu}\right) N, \\
\mathcal{L}_{\rho K K}=i g_{\rho K K}\left(K \rho^{\mu} \partial_{\mu} \bar{K}-\partial_{\mu} K \rho^{\mu} \bar{K}\right) .
\end{gathered}
$$

In the above, $\sigma^{\mu \nu}=i\left[\gamma^{\mu}, \gamma^{\nu}\right] / 2$ with $\gamma^{\mu}$ denoting the Dirac gamma matrices; the isospin doublet kaon and nucleon field are denoted by $N$ and $K$, respectively; while the 


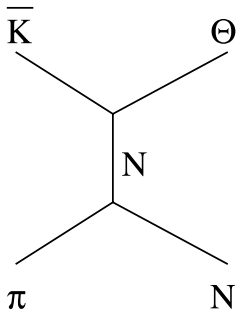

(1)

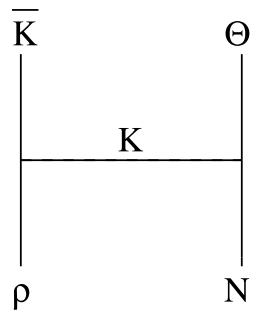

(3a)

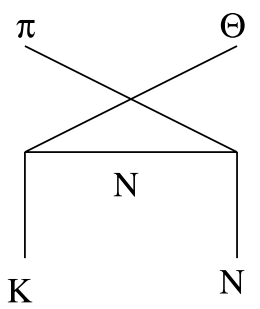

(2)

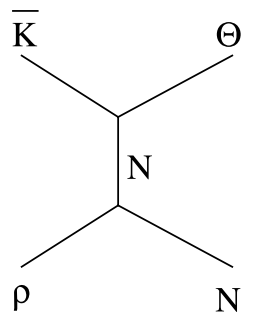

(3b)
FIG. 1. Diagrams for $\Theta^{+}$production from meson-nucleon reactions.

isospin triplet pion and $\rho$ meson fields are given by $\pi$ $=\vec{\tau} \cdot \vec{\pi}$ and $\rho^{\mu}=\vec{\tau} \cdot \vec{\rho}^{\mu}$, respectively, with $\vec{\tau}$ denoting the Pauli spin matrices. The spin $1 / 2$ and isospin 0 pentaquark baryon is denoted by the $\Theta$ field. For coupling constants involving normal hadrons, they are taken to be $g_{\pi N N}$ $=13.5, g_{\rho N N}=3.25, g_{\rho K K}=3.25, \kappa_{\rho}=6.1$ as usually used in hadronic models [16]. The coupling constant $g_{K N \Theta}$ between $\Theta^{+}$and $N K$ is determined from its width given by

$$
\Gamma_{\Theta}=\frac{g_{K N \Theta}^{2}}{2 \pi} \frac{k\left(\sqrt{m_{N}^{2}+k^{2}}-m_{N}\right)}{m_{\Theta}},
$$

where $m_{N}$ and $m_{\Theta}$ are the masses of nucleon and $\Theta^{+}$, respectively, and $k$ is the center-of-mass momentum of $N$ and $K$ in the rest frame of $\Theta^{+}$. Using $m_{\Theta}=1.54 \mathrm{GeV}$ and $\Gamma_{\Theta}=20 \mathrm{MeV}$, we find $g_{K N \Theta}=4.4$, which is comparable to that given by the chiral soliton model [4].

The amplitudes for the three reactions shown in Fig. 1 are

$$
\begin{gathered}
\mathcal{M}_{1}=g_{\pi N N} g_{K N \Theta} \bar{\Theta}\left(p_{4}\right) \frac{m_{N}-p_{1}-p_{2}}{s-m_{N}^{2}} N\left(p_{2}\right), \\
\mathcal{M}_{2}=g_{\pi N N} g_{K N \Theta} \bar{\Theta}\left(p_{4}\right) \frac{m_{N}-p_{2}+p_{3}}{u-m_{N}^{2}} N\left(p_{2}\right), \\
\mathcal{M}_{3 a}=i g_{\rho K K} g_{K N \Theta} \bar{\Theta}\left(p_{4}\right) \gamma_{5} N\left(p_{2}\right) \frac{1}{t-m_{K}^{2}}\left(2 p_{3}-2 p_{1}\right)^{\mu} \epsilon_{\mu}, \\
\mathcal{M}_{3 b}=i g_{\rho N N} g_{K N \Theta} \bar{\Theta}\left(p_{4}\right) \gamma_{5} \frac{p_{1}+p_{2}+m_{N}}{s-m_{N}^{2}} \\
\times\left(\gamma^{\mu}-i \frac{\kappa_{\rho}}{2 m_{N}} \sigma^{\nu \mu} p_{1 \nu}\right) N\left(p_{2}\right) \epsilon_{\mu},
\end{gathered}
$$

where $\epsilon_{\mu}$ is the polarization vector of $\rho$ meson. In the

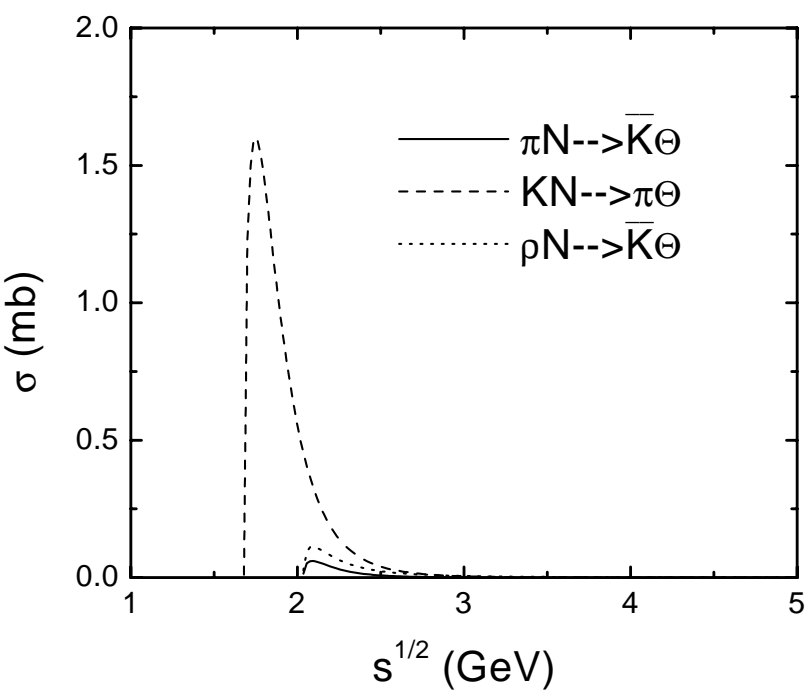

FIG. 2. Spin and isospin averaged cross sections for $\Theta^{+}$production from meson-nucleon reactions as functions of center-of-mass energy: $\pi N \rightarrow \bar{K} \Theta$ (solid curve), $K N \rightarrow \pi \Theta$ (dashed curve), and $\rho N$ $\rightarrow \bar{K} \Theta$ (dotted curve).

above, $p_{1}$ and $p_{2}$ denote the momenta of initial state particles while $p_{3}$ and $p_{4}$ denote those of final state particles on the left and the right side of a diagram. The usual Mandelstam variables are given by $s=\left(p_{1}+p_{2}\right)^{2}, t=\left(p_{1}\right.$ $\left.-p_{3}\right)^{2}$, and $u=\left(p_{1}-p_{4}\right)^{2}$.

The spin and isospin averaged cross sections for these reactions can be written as

$$
\frac{d \sigma}{d t}=\frac{1}{64 \pi s p_{i}^{2}} \frac{1}{S_{i}} \frac{1}{I_{i}} \sum_{s, I}\left|\mathcal{M}_{i}\right|^{2} .
$$

In the above, $S_{i}$ and $I_{i}$ denote spin and isospin factors, and their values are 2 and 6 for the reaction $\pi N \rightarrow \bar{K} \Theta, 2$ and 4 for the reaction $K N \rightarrow \pi \Theta$, and 6 and 6 for the reaction $\rho N \rightarrow \bar{K} \Theta$.

To evaluate the cross sections for these reactions, form factors are needed at interaction vertices to take into account the finite sizes of hadrons. We adopt the monopole form factor used in Refs. [17-20], i.e.,

$$
F\left(\mathbf{q}^{2}\right)=\frac{\Lambda^{2}}{\Lambda^{2}+\mathbf{q}^{2}},
$$

with $\mathbf{q}^{2}$ denoting either the squared three-momentum of external particles in $s$ and $u$ channel diagrams or the squared three-momentum transfer in $t$ channel diagrams. The cutoff parameter is taken to be $\Lambda=0.5 \mathrm{GeV}$ for all interaction vertices, based on fitting the measured cross section for the reaction $\pi N \rightarrow K \Lambda$ using similar hadronic Lagrangians [21].

The resulting spin and isospin averaged cross sections are shown in Fig. 2 as functions of center-of-mass energy. It is seen that all cross sections peak at energies slightly above their threshold. The largest cross section is from the reaction $K N \rightarrow \pi \Theta$, with peak value of about $1.6 \mathrm{mb}$ as result of small 


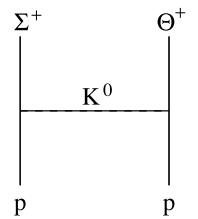

(4a)

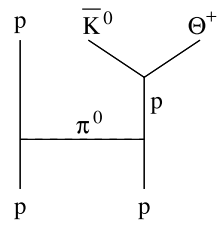

(6a)

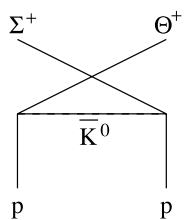

(4b)

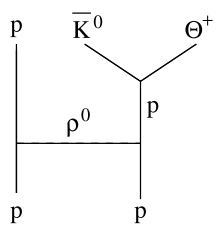

(6b)

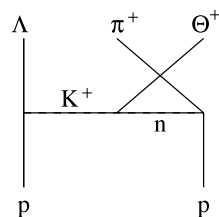

(5)

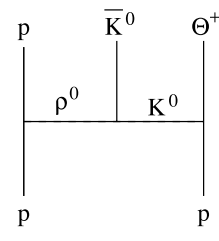

$(6 \mathrm{c})$
FIG. 3. Diagrams for $\Theta$ production from proton-proton reactions.

mass difference between initial and final states. The cross sections for other two reactions $\pi N \rightarrow \bar{K} \Theta$ and $\rho N \rightarrow \bar{K} \Theta$ are much smaller, with values about $0.05 \mathrm{mb}$ and $0.1 \mathrm{mb}$, respectively.

\section{III. @ PRODUCTION FROM PROTON-PROTON REACTIONS}

The $\Theta^{+}$baryon can also be produced from proton-proton reactions $p p \rightarrow \Sigma^{+} \Theta^{+}$with two-body final state as well as $p p \rightarrow \pi^{+} \Lambda \Theta^{+}$and $p p \rightarrow \bar{K}^{0} p \Theta^{+}$with three-body final states. These reactions are shown by the diagrams in Fig. 3. Additional interaction Lagrangians besides those given in Eq. (1) needed to evaluate their cross sections are

$$
\begin{gathered}
\mathcal{L}_{K N \Lambda}=i g_{K N \Lambda}\left(\bar{K} \bar{\Lambda} \gamma_{5} N+\bar{N} \gamma_{5} \Lambda \bar{K}\right), \\
\mathcal{L}_{K N \Sigma}=i g_{K N \Sigma} \bar{N} \gamma_{5} \bar{\Sigma} K+\text { H.c. }
\end{gathered}
$$

where $\Lambda$ and $\Sigma=\vec{\tau} \cdot \vec{\Sigma}$ denote the isospin singlet and triplet hyperons, respectively. The coupling constants $g_{K N \Lambda}$ and $g_{K N \Sigma}$ are related to $g_{\pi N N}$ via the $\mathrm{SU}(3)$ relations $[16,19]$ :

$$
\begin{gathered}
g_{K N \Lambda}=\frac{3-2 \alpha_{D}}{\sqrt{3}} g_{\pi N N} \simeq 13.5, \\
g_{K N \Sigma} \simeq\left(1-2 \alpha_{D}\right) g_{\pi N N}=-3.78,
\end{gathered}
$$

with $\alpha_{D}=D /(D+F)=0.64$.

For the reaction $p p \rightarrow \Sigma^{+} \Theta^{+}$with two-body final state, the two amplitudes are

$$
\begin{aligned}
& \mathcal{M}_{4 a}=-g_{K N \Theta} g_{K N \Sigma} \bar{\Sigma}\left(p_{3}\right) \gamma_{5} p\left(p_{1}\right) \frac{1}{t-m_{K}^{2}} \bar{\Theta}\left(p_{4}\right) \gamma_{5} p\left(p_{2}\right), \\
& \mathcal{M}_{4 b}=-g_{K N \Theta} g_{K N \Sigma} \bar{\Sigma}\left(p_{3}\right) \gamma_{5} p\left(p_{2}\right) \frac{1}{u-m_{K}^{2}} \bar{\Theta}\left(p_{4}\right) \gamma_{5} p\left(p_{1}\right) .
\end{aligned}
$$

Its cross section can be evaluated using Eq. (4) without isospin factor.
For the reactions $p p \rightarrow \pi^{+} \Lambda \Theta^{+}$and $p p \rightarrow \bar{K}^{0} p \Theta^{+}$with three-body final states, their amplitudes can be written in terms of those of off-shell two-body subprocesses involving the exchanged meson and the proton on the right side of a diagram $[19,20]$, i.e.,

$$
\begin{aligned}
& \mathcal{M}_{5}=i g_{K N \Lambda} \bar{\Lambda}\left(p_{3}\right) \gamma_{5} p\left(p_{1}\right) \frac{1}{t-m_{K}^{2}} \mathcal{M}_{K^{+} p \rightarrow \pi^{+} \Theta^{+}} \\
\mathcal{M}_{6}= & -i g_{\pi N N} \bar{p}\left(p_{3}\right) \gamma_{5} p\left(p_{1}\right) \frac{1}{t-m_{\pi}^{2}} \mathcal{M}_{\pi^{0} p \rightarrow \bar{K}^{0} \Theta^{+}}+g_{\rho N N} \bar{p}\left(p_{3}\right) \\
& \times\left[\gamma^{\mu}+i \frac{\kappa_{\rho}}{2 m_{N}} \sigma^{\alpha \mu}\left(p_{1}-p_{3}\right)_{\alpha}\right] p\left(p_{1}\right) \\
& \times\left[-g_{\mu \nu}+\frac{\left(p_{1}-p_{3}\right)_{\mu}\left(p_{1}-p_{3}\right)_{\nu}}{m_{\rho}^{2}}\right] \frac{1}{t-m_{\rho}^{2}} \mathcal{M}_{\rho^{0} p \rightarrow \bar{K}^{0} \Theta^{+}},
\end{aligned}
$$

where $\mathcal{M}_{K^{+} p \rightarrow \pi^{+} \Theta^{+}}, \mathcal{M}_{\pi^{0} p \rightarrow \bar{K}^{0} \Theta^{+}}$, and $\mathcal{M}_{\rho^{0} p \rightarrow \bar{K}^{0} \Theta^{+}}$are the amplitudes for the subprocesses $K^{+} p \rightarrow \pi^{+} \Theta^{+}, \quad \pi^{0} p$ $\rightarrow \bar{K}^{0} \Theta^{+}$, and $\rho^{0} p \rightarrow \bar{K}^{0} \Theta^{+}$. In terms of their off-shell cross sections, the cross sections for $\Theta^{+}$production in protonproton reactions with three-body final states are given by

$$
\begin{aligned}
\frac{d \sigma_{p p \rightarrow \pi^{+} \Lambda \Theta^{+}}}{d t d s_{1}}= & \frac{g_{K N \Lambda}^{2}}{32 \pi^{2} s p_{i}^{2}} k \sqrt{s_{1}}\left[-t+\left(m_{N}\right.\right. \\
& \left.\left.\quad-m_{\Lambda}\right)^{2}\right] \frac{F\left(\mathbf{q}^{2}\right)}{\left(t-m_{K}^{2}\right)^{2}} \sigma_{K^{+} p \rightarrow \pi^{+} \Theta^{+}}\left(s_{1}, t\right), \quad(11) \\
\frac{d \sigma_{p p \rightarrow \bar{K}^{0} p \Theta^{+}}}{d t d s_{1}}= & \frac{g_{\pi N N}^{2}}{32 \pi^{2} s p_{i}^{2}} k \sqrt{s_{1}}(-t) \frac{f(t)}{\left(t-m_{\pi}^{2}\right)^{2}} \sigma_{\pi^{0} p \rightarrow \bar{K}^{0} \Theta^{+}}\left(s_{1}, t\right) \\
& +\frac{3 g_{\rho N N}^{2}}{64 \pi^{2} s p_{i}^{2}} k \sqrt{s_{1}} \frac{f(t)}{\left(t-m_{\rho}^{2}\right)^{2}}\left[4\left(1+\kappa_{\rho}\right)^{2}(-t\right. \\
& \left.\left.-2 m_{N}^{2}\right)-\kappa_{\rho}^{2} \frac{\left(4 m_{N}^{2}-t\right)^{2}}{2 m_{N}^{2}}+4\left(1+\kappa_{\rho}\right) \kappa_{\rho}\left(4 m_{N}^{2}-t\right)\right] \\
& \times \sigma_{\rho^{0} p \rightarrow \bar{K}^{0} \Theta^{+}}\left(s_{1}, t\right) .
\end{aligned}
$$

In the above, $p_{i}$ is the center-of-mass momentum of two initial protons, $t$ is the squared four momentum transfer of exchanged meson, $s$ is the squared total center-of-mass energy, and $s_{1}$ and $k$ are, respectively, the squared invariant mass and center-of-mass momentum of exchanged meson in the subprocess. We note that there is no interference between amplitudes involving exchange of pion and $\rho$ meson.

For form factors at $\pi N N$ and $\rho N N$ vertices involving pion meson or $\rho$ meson exchange, we use the usual covariant monopole form factor [19]

$$
f(t)=\frac{\Lambda^{2}-m_{\mathrm{ex}}^{2}}{\Lambda^{2}-t}
$$

with $m_{\mathrm{ex}}$ denoting the mass of exchanged meson. The cutoff parameter $\Lambda$ is taken to have value $1.3 \mathrm{GeV}$ for pion 


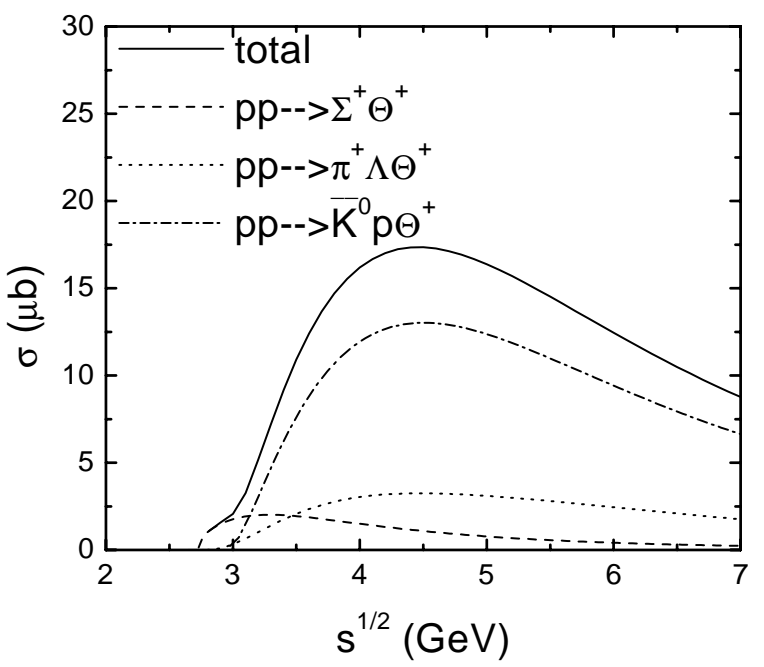

FIG. 4. Cross sections for $\Theta^{+}$production from proton-proton reactions $p p \rightarrow \Sigma^{+} \Theta^{+}$(dashed curve), $p p \rightarrow \pi^{+} \Lambda \Theta^{+}$(dotted curve), and $p p \rightarrow \bar{K}^{0} p \Theta^{+}$(dash-dotted curve) functions of center-of-mass energy. The total cross section is given by solid curve.

exchange and $1.4 \mathrm{GeV}$ for $\rho$ exchange as in Ref. [19]. At other interaction vertices, we use monopole form factors $F\left(\mathbf{q}^{2}\right)$ of Eq. (5) with same cutoff parameter $\Lambda=0.5 \mathrm{GeV}$ as introduced in Sec. II.

In Fig. 4, we show the cross sections for $\Theta^{+}$production from proton-proton reactions with either two-body or threebody final states as functions of center-of-mass energy. It is seen that the cross section for the reaction $p p \rightarrow \Sigma^{+} \Theta^{+}$ (dashed curve) with two-body final state has a peak value about $2 \mu \mathrm{b}$ around $3.3 \mathrm{GeV}$. This cross section is comparable to that for the reaction $p p \rightarrow \pi^{+} \Lambda \Theta^{+}$(dotted curve) with three-body final state, which is about $3 \mu \mathrm{b}$ at center-of-mass energy $4.2 \mathrm{GeV}$. These two cross sections are much smaller than the peak cross section of $13 \mu \mathrm{b}$ for the reaction $p p$ $\rightarrow \bar{K}^{0} p \Theta^{+}$(dash-dotted curve) with three-body final state. The total cross section for $\Theta^{+}$production from proton-proton reactions obtained from the sum of above three partial cross sections is shown by the solid curve. The $\Theta^{+}$production cross section from the reaction $p p \rightarrow \Sigma^{+} \Theta^{+}$has been previously estimated from the $n K^{+}$invariant mass spectrum in the reaction $p p \rightarrow n \Sigma^{+} K^{+}$using the phase-space argument with a
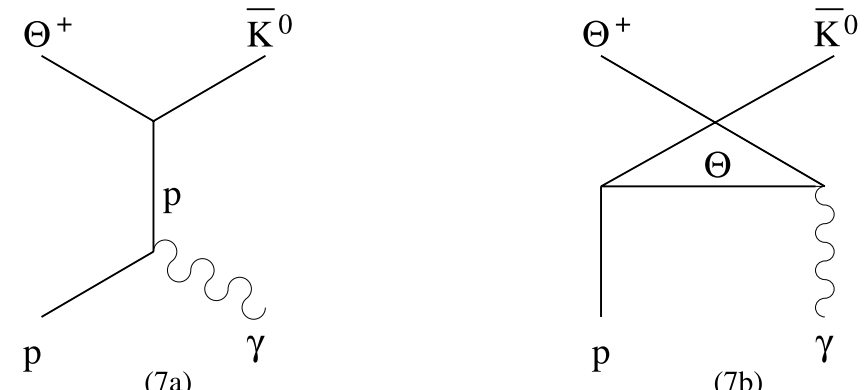

FIG. 5. Diagrams for $\Theta^{+}$production from photon-proton reactions with two-body final state.

$\Theta^{+}$width of $5 \mathrm{MeV}$ [22]. Its value at $100-200 \mathrm{MeV}$ above threshold is about $80-120 \mathrm{nb}$ and is about a factor of 20 smaller than our value. Part of the difference is due to the larger $\Theta^{+}$width of $20 \mathrm{MeV}$ used in our study for determining the coupling constant $g_{K N \Theta}$.

\section{IV. @ PRODUCTION FROM PHOTON-PROTON REACTIONS}

The hadronic Lagrangians introduced in previous sections can be generalized to include photons in order to evaluate the cross section for $\Theta^{+}$production from photon-proton reactions. This includes the reaction $\gamma p \rightarrow \bar{K}^{0} \Theta^{+}$with two-body final state as shown by the diagrams in Fig. 5 as well as the reactions $\gamma p \rightarrow K^{*-} \pi^{+} \Theta^{+}$and $\gamma p \rightarrow K^{-} \rho^{+} \Theta^{+}$with three-body final states as shown by the diagrams in Fig. 6 .

To evaluate the cross sections for these reactions, we need the following interaction Lagrangians involving photons [22]:

$$
\begin{gathered}
\mathcal{L}_{\gamma N N}=i e A^{\mu} \bar{N} \gamma_{\mu}\left[\left(1+\tau_{3}\right) / 2\right] N, \\
\mathcal{L}_{\gamma \Theta \Theta}=i e A^{\mu} \bar{\Theta} \gamma_{\mu} \Theta, \\
\mathcal{L}_{\gamma \pi \pi}=e A^{\mu}\left(\partial_{\mu} \vec{\pi} \times \vec{\pi}\right)_{3}, \\
\mathcal{L}_{\gamma \rho \rho}=e\left\{A^{\mu}\left(\partial_{\mu} \vec{\rho}^{\nu} \times \vec{\rho}_{\nu}\right)_{3}+\left[\left(\partial_{\mu} A^{\nu} \vec{\rho}_{\nu}-A^{\nu} \partial_{\mu} \vec{\rho}_{\nu}\right) \times \vec{\rho}^{\mu}\right]_{3}\right. \\
\left.+\left[\vec{\rho}^{\mu} \times\left(A^{\nu} \partial_{\mu} \vec{\rho}_{\nu}-\partial_{\mu} A^{\nu} \vec{\rho}_{\nu}\right)\right]_{3}\right\},
\end{gathered}
$$
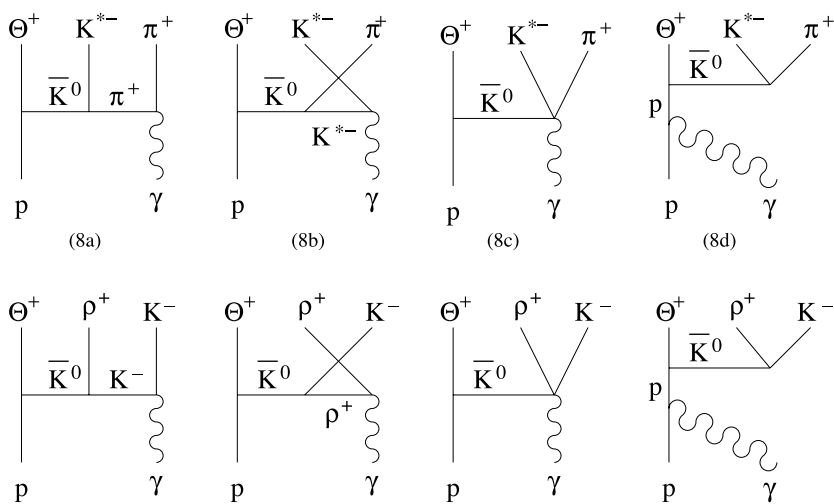

(9a)

(9b)

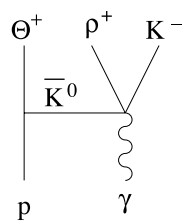

$(9 \mathrm{c})$

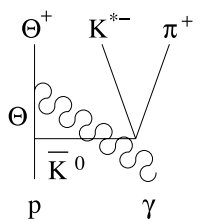

(8e)

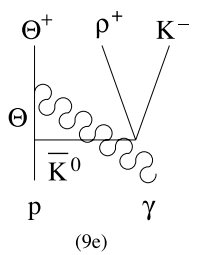

FIG. 6. Diagrams for $\Theta^{+}$production from photon-proton reactions with three-body final states. 


$$
\begin{gathered}
\mathcal{L}_{\gamma K K}=i e A^{\mu}\left[K Q \partial_{\mu} \bar{K}-\partial_{\mu} K Q \bar{K}\right], \\
\mathcal{L}_{\gamma \pi K K^{*}}=-e g_{\pi K K^{*}} A^{\mu}\left(K_{\mu}^{*}(2 \vec{\tau} Q-Q \vec{\tau}) \bar{K}+K(2 Q \vec{\tau}\right. \\
\left.-\vec{\tau} Q) \bar{K}_{\mu}^{*}\right) \cdot \vec{\pi}, \\
\mathcal{L}_{\gamma \rho K K}=e g_{\rho K K} A^{\mu} K(\vec{\tau} Q+Q \vec{\tau}) \bar{K} \cdot \bar{\rho}_{\mu},
\end{gathered}
$$

where $A_{\mu}$ denotes the photon field and $Q$ is the diagonal charge operator with elements 0 and -1 . Also needed is the interaction Lagrangian between $\pi$ and $K K^{*}$, which is given by

$$
\mathcal{L}_{\pi K K^{*}}=i g_{\pi K K^{*}} K^{* \mu}\left(\bar{K} \partial_{\mu} \pi-\partial_{\mu} \bar{K} \pi\right)+\text { H.c. },
$$

with the coupling constant $g_{\pi K K^{*}}=3.28$ determined from the decay width of $K^{*}$.

For the reaction $\gamma p \rightarrow \bar{K} \Theta^{+}$with two-body final state, its amplitude is

$$
\mathcal{M}_{7}=\mathcal{M}_{7 a}+\mathcal{M}_{7 b}
$$

with

$$
\begin{gathered}
\mathcal{M}_{7 a}=-e g_{K N \Theta} \frac{1}{s-m_{N}^{2}} \bar{\Theta}\left(p_{3}\right) \gamma^{5}\left(p_{1}+p_{2}+m_{N}\right) \gamma^{\mu} N\left(p_{1}\right) \epsilon_{\mu}, \\
\mathcal{M}_{7 b}=-e g_{K N \Theta} \frac{1}{u-m_{\Theta}^{2}} \bar{\Theta}\left(p_{3}\right) \gamma^{\mu}\left(p_{1}-p_{4}+m_{\Lambda_{c}}\right) \gamma^{5} N\left(p_{1}\right) \epsilon_{\mu},
\end{gathered}
$$

where $\epsilon_{\mu}$ is the polarization vector of $\gamma$.

For form factors, an overall one is multiplied to the total amplitude of each reaction in order to maintain gauge invariance for the resulting amplitude, as in Ref. [20] for photoproduction of charmed hadrons on protons. This form factor is taken to have the monopole form of Eq. (5) but with $\mathbf{q}$ denoting the photon three-momentum in center-of-mass system. As in photoproduction of charmed hadrons, we use a cutoff parameter $\Lambda=0.75 \mathrm{GeV}$. The cross section for the reaction $\gamma p \rightarrow K^{0} \Theta^{+}$with two-body final state can be similarly written as that of Eq.(4) without isospin factor.

For the reactions $\gamma p \rightarrow K^{-} \pi^{+} \Theta^{+}$and $\gamma p \rightarrow K^{-} \rho^{+} \Theta^{+}$with three-body final states via $\bar{K}^{0}$ exchange, the photon couples either to $K$ meson as in first three diagrams or to external baryons as in other two diagrams. Since the contributions from latter diagrams are much smaller than those from the former diagrams, as shown explicitly in charmed hadron production from proton-proton reactions with three-body final states [20], they are thus neglected in following calculations. As a result, results obtained in the present study for $\Theta^{+}$production with three-body final states violate slightly the gauge invariance. The amplitudes for the two reactions with threebody final states can then be written as

$$
\mathcal{M}_{i}=i g_{K N \Theta} \bar{\Theta}\left(p_{3}\right) \gamma_{5} N\left(p_{1}\right) \frac{1}{t-m_{K}^{2}}\left(\mathcal{M}_{i a}^{s u b}+\mathcal{M}_{i b}^{s u b}+\mathcal{M}_{i c}^{s u b}\right),
$$

with $i=8$ and 9 denoting, respectively, the reaction $\gamma p$ $\rightarrow K^{-} \pi^{+} \Theta^{+}$and the reaction $\gamma p \rightarrow K^{-} \rho^{+} \Theta^{+}$in Fig. 6. In the above, the amplitudes $\mathcal{M}_{i a}^{\text {sub }}, \mathcal{M}_{i b}^{\text {sub }}$, and $\mathcal{M}_{i c}^{\text {sub }}$ are for the subprocesses $\gamma \bar{K}^{0} \rightarrow \pi^{+} K^{*-}$ and $\gamma \bar{K}^{0} \rightarrow \rho^{+} K^{-}$, and they are given explicitly by

$$
\begin{aligned}
M_{8 a}^{s u b}= & \sqrt{2} e g_{\pi K K^{*}}\left(-2 k_{1}+k_{3}\right)^{\mu} \frac{1}{\left(k_{1}-k_{3}\right)^{2}-m_{K}^{2}}\left(k_{1}-k_{3}\right. \\
& \left.+k_{4}\right)^{v} \varepsilon_{3 \mu} \varepsilon_{2 \nu},
\end{aligned}
$$

$$
\begin{aligned}
M_{8 b}^{s u b}= & -\sqrt{2} e g_{\pi K K^{*}}\left(-k_{1}-k_{4}\right)^{\alpha} \frac{1}{\left(k_{1}-k_{4}\right)^{2}-m_{K^{*}}^{2}}\left[g_{\alpha \beta}\right. \\
& \left.-\frac{\left(k_{1}-k_{4}\right)_{\alpha}\left(k_{1}-k_{4}\right)_{\beta}}{m_{K^{*}}^{2}}\right] \times\left[\left(-k_{2}-k_{3}\right)^{\beta} g^{\mu \nu}\right. \\
& \left.+\left(-k_{1}+k_{2}+k_{4}\right)^{\nu} g^{\beta \mu}+\left(k_{1}+k_{3}-k_{4}\right)^{\mu} g^{\beta \nu}\right] \varepsilon_{3 \mu} \varepsilon_{2 \nu},
\end{aligned}
$$

$$
M_{8 c}^{s u b}=\sqrt{2} e g_{\pi K K^{*}} g^{\mu \nu} \varepsilon_{3 \mu} \varepsilon_{2 \nu},
$$

$$
\begin{aligned}
M_{9 a}^{s u b}= & \sqrt{2} e g_{\rho K K}\left(-2 k_{1}+k_{3}\right)^{\mu} \frac{1}{\left(k_{1}-k_{3}\right)^{2}-m_{K}^{2}}\left(k_{1}-k_{3}\right. \\
& \left.+k_{4}\right)^{\nu} \varepsilon_{3 \mu} \varepsilon_{2 \nu},
\end{aligned}
$$

$$
\begin{gathered}
M_{9 b}^{\text {sub }}=-\sqrt{2} e g_{\rho K K}\left(-k_{1}-k_{4}\right)^{\alpha} \frac{1}{\left(k_{1}-k_{4}\right)^{2}-m_{\rho}^{2}}\left[g_{\alpha \beta}\right. \\
\left.-\frac{\left(k_{1}-k_{4}\right)_{\alpha}\left(k_{1}-k_{4}\right)_{\beta}}{m_{\rho}^{2}}\right] \times\left[\left(-k_{2}-k_{3}\right)^{\beta} g^{\mu \nu}\right. \\
\left.+\left(-k_{1}+k_{2}+k_{4}\right)^{\nu} g^{\beta \mu}+\left(k_{1}+k_{3}-k_{4}\right)^{\mu} g^{\beta \nu}\right] \varepsilon_{3 \mu} \varepsilon_{2 \nu}, \\
M_{9 c}^{\text {sub }}=\sqrt{2} e g_{\rho K K} g^{\mu \nu} \varepsilon_{3 \mu} \varepsilon_{2 \nu} .
\end{gathered}
$$

In the above, $k_{1}$ and $k_{4}$ are the momenta of initial $K$ and final pseudoscalar meson, while $k_{2}$ and $k_{3}$ are those of initial photon and final vector meson with their polarization vectors denoted by $\epsilon_{2}$ and $\epsilon_{3}$, respectively.

As in proton-proton reactions, cross sections for the two reactions $\gamma p \rightarrow K^{*-} \pi^{+} \Theta^{+}$and $\gamma p \rightarrow K^{-} \rho^{+} \Theta^{+}$with three-body final states can be expressed in terms of off-shell cross sections for the subprocesses $\gamma \bar{K}^{0} \rightarrow K^{*-} \pi^{+}$and $\gamma \bar{K}^{0} \rightarrow \rho^{+} K^{-}$in- 


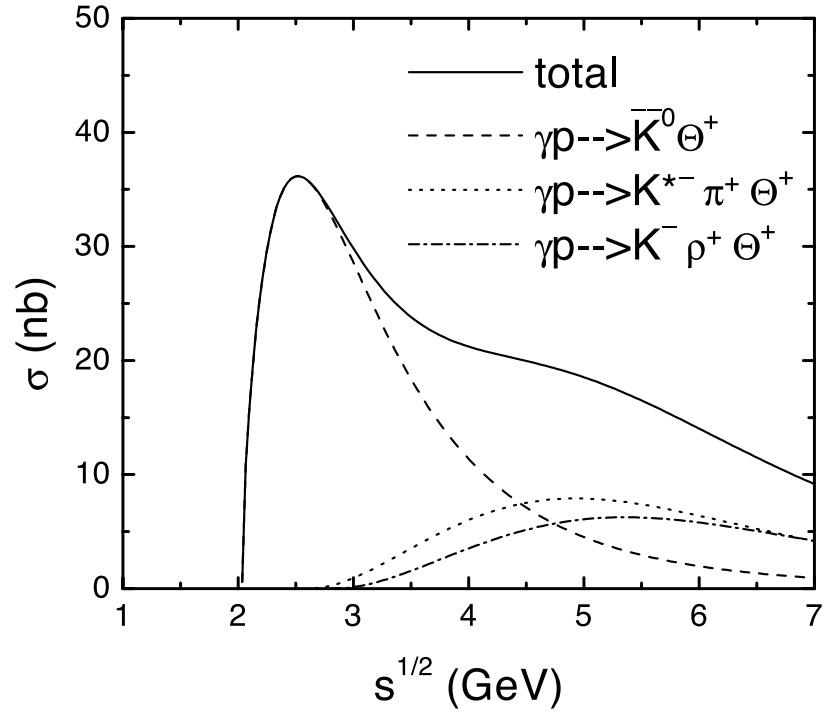

FIG. 7. Cross sections for $\Theta^{+}$production from photon-proton reactions as functions of center-of-mass energy: total (solid curve), $\gamma p \rightarrow \bar{K}^{0} \Theta^{+}$(dashed curve), $\gamma p \rightarrow K^{*-} \pi^{+} \Theta^{+}$(dotted curve), and $\gamma p$ $\rightarrow K^{-} \rho^{+} \Theta^{+}$(dash-dotted curve).

volving two particles in final states, i.e.,

$$
\begin{aligned}
\frac{d \sigma_{\gamma p \rightarrow K^{*-} \pi^{+} \Theta^{+}}}{d t d s_{1}}= & \frac{g_{K N \Theta}^{2}}{32 \pi^{2} s p_{i}^{2}} k \sqrt{s_{1}}\left[-t+\left(m_{N}\right.\right. \\
& \left.\left.-m_{\Theta}\right)^{2}\right] \frac{F\left(\mathbf{q}^{2}\right)}{\left(t-m_{K}^{2}\right)^{2}}\left[\sigma_{\gamma K^{0} \rightarrow K^{*-} \pi^{+}}\left(s_{1}, t\right)\right], \\
\frac{d \sigma_{\gamma p \rightarrow K^{-} \rho^{+} \Theta^{+}}=}{d t d s_{1}}= & \frac{g_{K N \Theta}^{2}}{32 \pi^{2} s p_{i}^{2}} k \sqrt{s_{1}}\left[-t+\left(m_{N}\right.\right. \\
& \left.\left.-m_{\Theta}\right)^{2}\right] \frac{F\left(\mathbf{q}^{2}\right)}{\left(t-m_{K}^{2}\right)^{2}}\left[\sigma_{\gamma \bar{K}^{0} \rightarrow \rho^{+} K^{-}}\left(s_{1}, t\right)\right],
\end{aligned}
$$

where $p_{i}, k$, and $s$ are similarly defined as in Eq. (12) for proton-proton reactions with three-body final states. The form factor $F\left(\mathbf{q}^{2}\right)$ at $K N \Theta$ vertex is taken to have the same form in Eq. (5) with the cutoff parameter $\Lambda=0.5 \mathrm{GeV}$ as used in $\Theta^{+}$production from meson-proton and protonproton reactions. Furthermore, we have introduced an overall monopole form factor for two-body subprocesses $\gamma \bar{K}^{0} \rightarrow K^{*-} \pi^{+}$and $\gamma \bar{K}^{0} \rightarrow \rho^{+} K^{-}$with the same cutoff parameter $\Lambda=0.75 \mathrm{GeV}$ such as that used in charmed hadron production from photon-proton reactions [21].

In Fig. 7, we show the cross sections for $\Theta^{+}$production from photon-proton reactions. It is seen that the cross section with two-body final state, i.e., $\gamma p \rightarrow \bar{K}^{0} \Theta^{+}$(dashed curve), dominates at low center-of-mass energies with peak value about $36 \mathrm{nb}$, while those with three-body final states are more important at high center-of-mass energies with maximum value of $8 \mathrm{nb}$ for $\gamma p \rightarrow K^{*-} \pi^{+} \Theta^{+}$(dotted curve) and $6 \mathrm{nb}$ for $\gamma p \rightarrow K^{-} \rho^{+} \Theta^{+}$(dash-dotted curve). The total cross section including both two-body and three-body final states is given by the solid curve.

\section{SUMMARY}

The cross sections for the production of $\Theta^{+}$baryon consisting of $u u d d \bar{s}$ quarks from meson-nucleon, proton-proton, and photon-proton reactions are evaluated in a hadronic model that includes the $K N \Theta$ interaction with coupling constant determined from the width of $\Theta^{+}$. This model is based on a gauged SU(3) flavor symmetric Lagrangian with the photon introduced as a $\mathrm{U}_{\mathrm{em}}(1)$ gauged particle. Symmetry breaking effects are taken into account by using empirical hadron masses and coupling constants. Form factors of monopole type are introduced at interaction vertices to take into account finite hadron sizes, and values of the cutoff parameters are taken from fitting known cross sections of other reactions based on similar hadronic models. It is found that for meson-nucleon reactions, i.e., $\pi N \rightarrow \bar{K} \Theta^{+}, K N$ $\rightarrow \pi \Theta^{+}$, and $\rho N \rightarrow \bar{K} \Theta^{+}$, the one induced by kaon has the largest cross section of about $1.5 \mathrm{mb}$ and is almost an order of magnitude larger than those for reactions induced by pion and $\rho$ meson. For proton-proton reactions, the total cross section is about $20 \mu \mathrm{b}$ and is dominated by the reaction $p p$ $\rightarrow \bar{K}^{0} p \Theta^{+}$with only about $25 \%$ from the reactions $p p$ $\rightarrow \Sigma^{+} \Theta^{+}$and $p p \rightarrow \pi^{+} \Lambda \Theta^{+}$. In photon-proton reactions, the reaction $\gamma p \rightarrow \bar{K}^{0} \Theta^{+}$with two-body final state is most important near threshold, and its value is about $36 \mathrm{nb}$. At higher energy, the reactions $\gamma p \rightarrow K^{*-} \pi^{+} \Theta^{+}$and $\gamma p \rightarrow K^{-} \rho^{+} \Theta^{+}$with three-body final states become important with comparable cross sections of about $10 \mathrm{nb}$. Knowledge on these cross sections is useful for studying $\Theta^{+}$production not only in elementary reactions involving hadrons and photons but also in relativistic heavy ion collisions, where final hadronic effects on $\Theta^{+}$production and absorption need to be understood in order to infer its production from the initial quark-gluon plasma.

\section{ACKNOWLEDGMENTS}

We thank Gerald A. Miller and Kazuo Tsushima for comments on an earlier version of the paper. This paper was based on work supported in part by the U.S. National Science Foundation under Grant No. PHY-0098805 and the Welch Foundation under Grant No. A-1358. 
[1] T. Nakano et al., Phys. Rev. Lett. 91, 012002 (2003).

[2] S. Stepanyan et al., CLAS Collaboration, hep-exp/0307018.

[3] V. V. Barmin et al., hep-ex/0304040.

[4] D. Diakonov, V. Petrov, and M. Poliakov, Z. Phys. A 359, 305 (1997).

[5] Fl. Stancu and D. O. Riska, hep-ph/0307010.

[6] M. Karliner and H. J. Lipkin, hep-ph/0307243.

[7] S. L. Zhu, hep-ph/0307042.

[8] C. E. Garlson, C. D. Carone, H. J. Kwee, and V. Nazaaryan, hep-ph/0307396.

[9] X. Chen, Y. Mao, and B. Q. Ma, hep-ph/0307381.

[10] J. Randrup, nucl-th/0307042.

[11] C. Adler et al., STAR Collaboration, Phys. Rev. Lett. 89, 092301 (2002).

[12] K. Adcox et al., PHENIX Collaboration, Phys. Rev. Lett. 89, 092302 (2002).
[13] L. W. Chen, V. Greco, C. M. Ko, S. H. Lee, and W. Liu, nucl-th/0308006.

[14] R. A. Arndt, I. I. Strakovsky, and R. L. Workman, hep-ph/ 0308012.

[15] J. Rafelski and B. Müller, Phys. Lett. B 101, 111 (1982); P. Koch, B. Müller, and J. Rafelski, Phys. Rep. 142, 167 (1986).

[16] C. H. Li and C. M. Ko, Nucl. Phys. A712, 110 (2002).

[17] Z. Lin, C. M. Ko, and B. Zhang, Phys. Rev. C 61, 024904 (2000).

[18] Z. Lin, T. G. Di, and C. M. Ko, Nucl. Phys. A689, 965 (2001).

[19] W. Liu and C. M. Ko, Phys. Lett. B 533, 259 (2002); W. Liu, C. M. Ko, and Z. W. Lin, Phys. Rev. C 65, 015203 (2002).

[20] W. Liu, S. H. Lee, and C. M. Ko, Nucl. Phys. A724, 375 (2003).

[21] W. Liu, C. M. Ko, and S. H. Lee, Nucl. Phys. (in press); nucl-th/0308013.

[22] M. V. Polyakov, A. Sibirtsev, K. Tsushima, W. Cassing, and K. Goeke, Eur. Phys. J. A 9, 115 (2000). 